Ann. Abeille, Ig64,

\title{
L'INFLUENCE DES FACTEURS EXTERNES SUR LA PRODUCTION DU NECTAR
}

\author{
R. W. SHUEL \\ Department of Apiculture, Ontario Agricultural College, \\ Guelph, Ontario (Canada)
}

\section{SOMMAIRE}

L'auteur passe en revue les travaux récents qui ont été publiés sur l'influence des facteurs externes (radiation solaire, température de l'air, l'humidité atmosphérique, fertilité du sol) sur la production de nectar par les plantes. Il conclut sur les possibilités d'améliorer cette production par les méthodes culturales, dans le cas où il s'agit de plantes cultivées.

Comme toutes les autres recherches, celles qui concernent la sécrétion nectarifère peuvent être effectuées pour deux raisons distinctes, soit pour satisfaire notre curiosité intellectuelle soit pour acquérir des connaissances susceptibles d'applications pratiques. Les recherches qui sont poursuivies dans le premier de ces buts sont généralement de nature théorique; ce sont des recherches de base. Celles qui sont conduites dans le second but peuvent être théoriques ou "appliquées ". Paradoxalement, les recherches entreprises sans motif spécifique autre que la solution d'une intéressante énigme se révèlent souvent être d'un grand intérêt pratique.

J'estime que les recherches sur la sécrétion nectarifère doivent être des recherches de base dans la mesure où le chercheur les rattache à la physiologie végétale. Bien des aspects physiologiques, autres que celui de la secrétion, peuvent être inclus dans le processus. Le milieu peut influencer l'approvisionnement en constituants utilisables pour la sécrétion du nectar plutôt que le processus de sécrétion en soi. Il est souvent difficile de faire la distinction entre effets directs et effets indirects et, pour cette raison, le terme " production du nectar » a été utilisé dans le titre de cette revue plutôt que celui de "sécrétion du nectar ». La floraison est un des derniers événements

(1) Rapport présenté au XIXe Congrès international d'Apiculture, Prague r963, Colloque sur la sécrétion nectarifère. 
R. W. SHUEL,

qui intervienne dans la vie d'une plante (d'une plante annuelle au moins) et tout facteur auquel le végétal a été soumis avant sa floraison aura, à quelque degré, une influence sur celle-ci et, avec elle, sur la production du nectar. Dans ce rapport, j'essaierai d'examiner l'action des facteurs du milieu qui concernent : la photosynthèse et l'approvisionnement en constituants organiques du nectar, la croissance, y compris la croissance avant et pendant la floraison ainsi que le rapport des croissances végétative et reproductive et, enfin le processus sécrétoire proprement dit.

INFLUENCE DES FACTEURS CLIMATIQUES SUR IA PRODUCTIOA DE NECTAR

Radiation solaire.

L'effet défavorable sur la sécrétion nectarifère de toute restriction dans l'approvisionnement des nectaires en hydrates de carbone a été amplement démontré (Von Czarnowski, I952 ; Girnik, I960; Wykes, I952). Des relevés de récoltes du miel portant sur de longues périodes ont montré qu'un temps clair est important pour cette production (Jorgensen et Markham, I953; Kexoyer, I9I7; MOFFET et Parker, I953). Pedersen (I953) a montré que la lumière solaire est le facteur du milieu le plus important dans le déterminisme de la production du nectar par la luzerne (Medicago sativa). Un rapport étroit a été trouvé entre l'énergie solaire incidente et la production de nectar chez Trifolium pratense (SHUEL, I952) Antirrhinum majus et Trifolium hybridum (SHUEr, I955, I959) ainsi que Tilia sp. (SiRnIK, I960). Les variations dans la production de nectar en fonction de l'irradiation peuvent être de l'ordre de plusieurs centaines pour cent (ShuEL, I952).

I1 n'est pas toujours possible de prévoir le caractère immédiat de l'influence de la lumière solaire sur la production du nectar. Les précurseurs immédiats des sucres du nectar peuvent être aussi bien des sucres récemment synthétisés que des hydrates de carbone de réserve (I)Aumann, I935). Percival, (I946) n'a pas observé d'effet de la variation de l'insolation sur la production journalière du nectar chez Rubus fruticosus, ce qui suppose que chez cette plante le nectar dérive d'hydrates de carbone de réserve. La même remarque peut être faite à propos du travail de MAKsrmiuk sur le Tilleul; dans ce cas, l'insolation pendant la période de secrétion ne semble amener que peu de différences dans la production du nectar (Maksymiuk, Ig60). Ces résultats, en ce qui concerne le Tilleul, sont en contradiction avec ceux de Girsik (Ig60).

Les mêmes considérations s'appliquent au nectar sécrété par les fleurs des arbres fruitiers dans les régions tempérées. Ces végétaux fleurissent et sécrètent leur nectar avant que les feuilles ne soient pleinement développées.

L'optimum d'énergie solaire peut varier largement selon les espèces. Généralement, l'intensité de la radiation requise pour un maximum de photosynthèse sur la surface totale des feuilles d'une plante est beaucoup plus élevée proportionnellement que pour une feuille isolée, par suite de l'effet d'ombre (MAURizio, I954). L'influence favorable sur la production totale de nectar d'une plantation à large espacement (GlushKov, I958 ; KazIEV, I958 ; PEDERSEN, r957) peut être due, dans une large mesure, à l'exposition d'une surface relativement grande de feuillage à la lumière directe du soleil. 
Température de l'air.

La température a été plus étudiée que tout autre facteur. Il existe des différences d'opinion en ce qui concerne son importance. BARBIER (I952) la considère comme de première importance pour l'Eucalyptus ; par contre, BEUTLER (I930) ne lui accorde qu'un intérêt mineur.

L'analyse d'enregistrements du poids des ruches effectuée aux États-Unis a révélé une corrélation directe entre production du miel et température (JORGENSEN et MARKHAM, I953). KIERULF (I957) et UKkELBERG (I960) ont enregistré des récoltes supérieures de miel de Bruyère dans les années où prévalent des températures relativement hautes. Il existe de nombreuses publications indiquant les marges de température favorables, lesquelles varient d'ailleurs avec les espèces (DEMIANowICz et Hiyx, I960 ; Glushkov, I958; Huber, I956 ; Jablonski, I960; Jaroszynska I960; KAZIEV, I96I).

Il est extrêmement difficile d'isoler les effets de la température, particulièrement dans les études sur le terrain. Les enregistrements de la température diurne, par exemple, peuvent refléter des conditions d'insolation qui, en soi, causent de larges variations de la miellée. Les enregistrements de température nocturne (PONOMAREVA et BukHAREVA, I958) sont moins équivoques.

DEmuth (1923) a observé que chez Tilia americana la sécrétion du nectar est liée à un seuil de température d'environ ${ }^{\circ} 8^{\circ}$. Il est probable que les températures minima critiques varient considérablement avec la zone climatique à laquelle les espèces sont adaptées. Les seuils de température, en ce qui concerne la sécrétion du nectar, peuvent fort bien représenter les températures d'activation des systèmes enzymatiques qui sont certainement mis en jeu dans le processus de sécrétion (BROWN, I959; Frey-WYSSLiNG et al., I954; SHUEL, I957; ZIEGLER et LüTTGE, I955; ZIMMERMANN, I953).

Le microclimat à l'intérieur de la fleur peut être important pour la sécrétion. La température à l'intérieur de la fleur peut être plus élevée de plusieurs degrés que celle de l'air ambiant (BüDEr, I959).

\section{Humidité atmosphérique.}

A l'exception du travail de Pankratova (citée par BEUTLER, I953) sur l'augmentation de la sécrétion des sucres chez Lythrum salicaria en atmosphère très humide, il n'y a que peu d'éléments permettant de penser que l'humidité affecte de façon appréciable la sécrétion nectarifère. Les variations post-sécrétoires dans le nectar sous 1'influence d'échanges de molécules de vapeur d'eau avec l'atmosphère sont par contre banales (SHUEL, I955) ; ces variations peuvent modifier l'accessibilité et l'attractivité de la plante pour les insectes (BUTLER, I945).

\section{INFLUENCE DES FACTEURS LIÉS AU SOL}

Les facteurs liés au sol sont d'un intérêt pratique en ce sens qu'ils sont susceptibles d'être contrôlés à un degré qui n'est pas accessible en ce qui concerne les facteurs climatiques. Un approvisionnement du sol en eau convenant à une bonne croissance végétative est une condition nécessaire à la réalisation d'une bonne saison 
de miel. HEIDEMANN (I958) considère que si mai et juin sont humides les conditions sont favorables à une bonne miellée de Bruyère. Il ressort d'une étude de KENOYER (I9I7) basée sur trente années de relevés de production de miel que les meilleures saisons en Iowa sont sensiblement plus humides que la moyenne et sont précédées par des périodes où les précipitations sont supérieures à la moyenne. BuLAxov (I958) tire les mêmes conclusions pour la miellée du Tilleul. Czarnowskr (I953) a trouvé que la production de nectar et celle de fleurs sont réduites chez le Bourrache croissant en sol sec.

En plus de leur influence à long terme sur la sécrétion du nectar via la croissance, les conditions physiques du sol peuvent affecter plus directement la sécrétion du nectar. Shuer, et Shivas (r953) ont exposé des Mufliers à différentes conditions de sol au moment de la floraison. Une déviation dans un sens ou dans l'autre du niveau optimum des conditions de croissance (field capacity) a réduit la sécrétion nectarifère. Le nectar était relativement beaucoup plus concentré aussi bien en sol très humide qu'en sol très sec. Des températures du sol comprises entre $2 \mathrm{I}^{\circ}$ et $27^{\circ}$ étaient supérieures à une température de $I 6^{\circ}$. Des restrictions dans 1'aération du sol réduisaient le sucre total du nectar mais accroissait la concentration. Aucune explication n'a pu être offerte de ce phénomène.

\section{Fertilité du sol.}

Comme avec la plupart des autres facteurs, il est difficile d'affirmer que les modifications de la fertilité du sol ont une influence plus ou moins directe sur la sécrétion ou bien si elles agissent à travers les altérations de la croissance. Pour cette raison, les données expérimentales incluant la croissance, aussi bien que les mesures de nectar sont très utiles. Le nombre de fleurs produites peut être tout aussi significatif que la sécrétion de nectar par fleur. PEDERSEN (I953) a trouvé que l'attractivité de la luzerne pour les abeilles dépend de la quantité totale de nectar par plante plutôt que de la quantité moyenne par fleur individuelle.

L'action bénéfique des fumures, particulièrement avec phosphore et potassium a été rapportée pour différentes plantes (EWERT, I935; KAZIEV, I96I ; MONAKOVA, et Chebotnikova, I957; PLASS, I952). D'autres publications ont indiqué un effet négligeable sur la sécrétion nectarifère mais une augmentation de la production de fleurs (HASLER et MAURIZIO, I95I ; SchöNTAG, I952).

L'effet de différents éléments minéraux sur la croissance sont plus interdépendants qu'indépendants; la même interdépendance peut être envisagée pour la production du nectar. RYLE (I953) étudiant l'interaction de l'azote, du potassium et du phosphore conclut que lorsque le potassium est limitant pour la croissance, la production de nectar est faible et que lorsque la croissance est limitée par l'azote ou le phosphore un surplus relatif de potassium est bénéfique. HASLER et MAURIZIO (I95I) ont noté de faibles productions de nectar dans les sols déficients en potassium. Shuer, (r959) a trouvé une interaction du potassium et de phosphore en ce qui concerne la production du nectar, la croissance et la floraison chez le Muflier et le Trèfle violet. Les niveaux d'approvisionnement pour les deux éléments nécessaires à une production de nectar optimum étaient beaucoup plus élevés pour le Trèfle violet que pour le Muflier. L,e rapport potassium/phosphore règle l'expression de la croissance végétative, tandis qu'une forte teneur en phosphore assure la production des fleurs. 
Selon les expériences de RyLE, une limitation draconnienne de l'alimentation en potassium réduit la production de nectar par fleur. Au contraire un niveau élevé du phosphore réduit la sécrétion nectarifère unitaire. Il apparaît que les meilleures plantes nectarifères sont prođuites lorsqu'il y a un équilibre raisonnable entre les deux éléments.

Un effet important d'une teneur élevée en potassium, jusqu'ici inexpliqué, est une réduction de la concentration du nectar (FWERT, I935; SHAw et al., I957; SHUEI, I959).

Une croissance végétative excessive provoquée par un excès d'azote est défavorable à la production du nectar (RYI,E, I953; SHUEL, I955); particulièrement par temps couvert (SHUEL, I955). Dès I846, LIEBIG avançait l'hypothèse que le nectar représente une excrétion en réponse à une concentration en sucres trop élevée par rapport à l'azote de la plante.

De bonnes miellées ont été signalées chez les plantes croissant sur un sol calcaire, ce qui laisserait supposer que le calcium ou le magnésium ou le $\mathrm{pH}$ du sol peuvent affecter la sécrétion du nectar. CARLISLE et RYIE (I958) ont noté une augmentation de la production du nectar chez le 'Trèfle violet sous l'influence du chauffage ; cet effet n'était apparemment pas associé au pH du sol. OER'TEI, (I93I) n'a pas trouvé de corrélation entre le $\mathrm{pH}$ du sol et la quantité de nectar sécrétée par le Trèfle blanc. Hasi,ER et Maurizio (I95I) n'ont pas obtenu d'amélioration de la production de nectar par application de magnésium à des parcelles de Brassica napus. La production du nectar chez le Trèfle violet poussant en cultures sur sable varie d'environ roo p. Ioo sur la base d'une influorescence et de plus de 300 p. Ioo sur la base d'une plante entière selon différentes combinaisons d'engrais calciques et magnésiens (SHUEL, I96I). Un niveau intermédiaire de calcium et un haut niveau de magnésium est apparemment sans effort ni avec le $\mathrm{pH}$ du sol ni avec un antagonisme des ions envers l'absorption des éléments majeurs. A ce propos, il est intéressant de noter que LüTTGE (Ig62) a trouvé un rapport magnésien/calcium anormalement élevé dans les nectaires.

Il semble établi que des applications de bore peuvent améliorer la sécrétion nectarifère dans less sols déficients en cet élément (HoLmes, I960).

Bien qu'il soit difficile de généraliser sur la base de données obtenues dans des conditions diverses, il se dégage d'elles un ensemble de conclusions possibles:

I) Les effets des fertilisants sur le nectar peuvent ne pas apparaître à l'échelle des grandes cultures mais ils n'en sont pas moins éminents dans les essais contrôlés à petite échelle. Ainsi, bien qu'ils soient probablement moins souvent limitants vis-à vis de la production du nectar que les facteurs climatiques, ils sont importants.

2) Leurs effets peuvent s'exercer aussi bien indirectement en modifiant la croissance des plantes que directement par modification de la sécrétion nectarifère. On ne sait que peu de chose encore à l'heure actuelle sur les deux modes d'action.

3) Les espèces peuvent varier considérablement dans leur optimum nectarifère en ce qui concerne aussi bien les qualités physiques du sol que les conditions de fertilité. En général, les conditions qui n'imposent pas de limitation appréciable à la croissance et qui assurent un équilibre raisonnable entre le développement végétatif et le développement reproducteur forment une base satisfaisante pour une bonne production de nectar. 


\section{L'AMÉLIORATION DE LA PRODUCTION DU NECTAR}

Il existe deux voies pour l'amélioration de la production du nectar, la voie génétique et la voie culturale. Nous considérerons seulement la seconde, car la première, bien que fort prometteuse (MaUrizio, I954; PEDERsen, I953 ; RyLE, I953) se situe hors du cadre de ce rapport.

D'utiles recommandations pour l'amélioration par les méthodes culturales pourraient être faites sur la base de nos connaissances actuelles mais il serait difficile de les mettre en pratique lorsque les sources de nectar ne sont pas sous le contrôle des apiculteurs. C'est la situation générale en Amérique du Nord, excepté dans les régions où le nectar est obtenu principalement de plantes cultivées pour les graines ou les fruits ; ces régions semblent bonnes aussi pour le nectar. Au contraire, les conditions favorables à la production du fourrage c'est-à-dire à la croissance végétative sont moins favorables au nectar.

Je voudrais pour terminer présenter quelques suggestions en ce qui concerne l'avenir des recherches sur la production du nectar:

I) $\mathrm{Il}$ y a bien des années, BonNier (1879) et plus tard Tschudin (I92I) ont remarqué que les plantes croissant en haute montagne sécrètent plus de nectar que celles qui, tout en appartenant à la même espèce, vivent près du niveau de la mer. Les plantes alpines sont caractérisées par leur petite taille et par le développement exagéré de leur système reproducteur.

2) Il serait intéressant de savoir si la teneur en auxine de ces plantes est plus, basse que celle des plantes de grande taille de la même espèce. Il est probable qu'une haute teneur en auxine réduit la sécrétion nectarifère (MATILE, I959; SHUEI, I959).

3) Durant ces dernières années, les physiologistes végétaux ont été intéressés par un nouveau groupe de substances régulatrices de la croissance et appelées " antigibbérellines ". Il a été récemment découvert que certains de ces composés abaissent la teneur en auxine des plantes (Kuraishi et Muir, I963).

Cette ligne de raisonnement, bien que hautement spéculative, est tentante. La possibilité d'influencer la secrétion du nectar par altération de la teneur en auxine des plantes mérite au moins d'être examinée.

Reçu pour publication en novembre 1963.

\section{SUMMARY}

INFLLENCE OF ENVIRONMENTAI, FACTORS ON NECTAR SECRETION

Work relating to the influence on nectar secretion of various environmental factors, including solar radiation, air temperature, atmospheric humidity, and soil factors, is reviewed. It is contended that research into nectar secretion should be related to the physiology of the plant, because many aspects of physiology in addition to the secretory process per se are involved in nectar production. Moreover, it is usually difficult if not impossible to state whether an observed effect of the environment is direct or indirect.

At present, certain generalizations can be made regarding conditions favoring high nectar production. It is suggested that research into the effects of growth-regulating compounds may reveal further possibilities for improving nectar production in cultivated plants. 


\section{RÉFÉRENCES BIBLIOGRAPHIQUES}

Barbier k. C., r952. Sécrétion de nectar chez les Eucalyptus. Rev. Fr. Apic., 3, I39-I45.

Bettler R., I930. Biologisch-chemische I'ntersuchungen am Nektar von Immenblumen. Z. vergl. Physiol., 12, $72-176$.

Beytler R., i953. Nertar. Bee World, 34, io6-i i6, i 28-136, I 56-162.

Boniter G., 1879. Les Nectaires. Ann. Sci. Nat., (Bot. Ser. VI), 8, 5-212.

Büdel A., I956. Das Mikroklima in einer Blüte. 2. Z. Bienenjorsch., 3, 185-190.

Bulanov N. A., I956. L'influence des précipitations sur la floraison et sur la production du nectar chez le tilleul (en russe ; Apic. Abstr. 192/58). Pchelovodstvo, 33, 33-34.

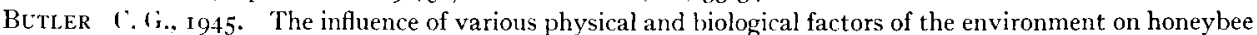
activity. An examination of the relationship between activity and nectar concentration and abundance. $J$. Exp. Biwl., 21, 5-12.

BRowx H. I)., 1959. Effects of respiratory inhibitors upon nectar secretion in Antirrhinum. Torrey Bull., 86, $290-205$.

CARLISLE E., RyLE M., 1958. The influence of nitrogen, phosphate, potash, and lime on the secretion of nectar by red clover in the field. Emp. J. Exp. Agric., 23, i 26-r 30.

Czarxowiki C. Von, 1952. Entersuchungen zur Frage der Nektarabsonderung. Z. Bienenforsch., 1, 1 $71-173$

Czarnowski C. Von, I953. Über die Einwirkung unterschiedlicher Wasserversorgung auf die Nektarsekretion beim Boretsch (Borago). Z. Bieneniorsch., 2, 85-91.

Daumanv E., I935. Die systematische Bedeutung des Blütennektariums der Gattung Iris. Bot. Zlb. Beih., Bi 53, 525-625.

Demianowicz Z., HLYN MI., 1960. Comparative studies of nectar secretion in seventeen varieties of lime. (En polonais.) Pcscel. Zess. Nauk., 4, i 33-г52.

Demtтi (;., 1923. Temperature and nectar secretion. Cleanings Bee Cull., 51, 582.

Ewert R., 1935. Die Förderung der Nektarabscheidung bei Raps, Bucheweizen, und Rotklee durch Kalidungung. Disch. Imkerjr., $9,63-66$.

Frey-Wyssuing A., Zimmermann M., Maurizio A., i954. Über den enzymatischen Zuckerumbau in Nektarien. Experientia, 10, 490-492.

Glusikov X. M., I958. Problems of beekeeping in the L. S. S. R. in relation to pollination. Bee World, 39, \$I-()2.

Girxik D. V., i958. Le processus de la sécrétion nectarifere chez le tilleul (en russe ; Apic. Abstr. z29i6o). Pchelorodstio, 35, 37-40.

Hasler A., Maurizio A., i95I. Über den Einfluss verschiedener Nahrstoffe auf Blutensatz, Nektarsekretion, und Samenertrag von honigenden Pflanzen, speziell von Sommerraps (Brassica napus I). Schweis. Bienenilg., 74, 208-219.

Heidenax, K., 1958. Ileidetracht, ein undefinierbarer Faktor in der Interei. Bienenbl. Bundesg., 11, $251-2.54$.

Holmes F. O., 1960. Boron deficiency as a probable cause of the failure of bees to visit certain flowers. Amer. Bée J., 100, 102-103.

Hüber H., I956. Die Abhängigkeit der Nektarsekretion von Temperature, Luft-und Bodenfeuchtigkeit. Planla, 48, $+7-98$.

Jablossk1 B., 196o. Der Einfluss der Saatdichte und verschiedener Aussaatzerten auf den Gebrauchwert von Phacelia tanacetitolia Benth. (en polonais). Pszczel. Zesz. Nauk., 4, 1-42.

GlowskA A., 1958. Comparative intensity of nectar secretion and bee activity on five varieties of fruit trees. (ell polonais). Pszcsel. Zesz. Nauk., 2, i 2 I-I 48.

Jaroszynska T., 1960. Floral biology and nectar secretion in Acer tataricum L. (en polonais). Pszczel. Zesz. Nauk., 4, I $53^{-1} 56$.

Jorgexsen C., Markiam F., 1953. Weather factors influencing honey production. Mich. Agr. Exp. Sta., Spec. Bull., 340.

KAZIEx T., I957. Production nectarifére et rendement du coton par différentes méthodes culturales (en russe ; Apic. Abstr. 33T/58). Pchelovodstoo, 34, 5 r-53.

Kaziev 'T., 1959. Einfluss klimatischer Bedingungen auf den Vorgang der Nektarsekretion bei Baumwolle (en russe; Apic. Abstr. 342/60). Pchelovodstzo, 36, 37-39.

Kaziev T., ig59 a. Einfluss von Düngern und Bewasserung auf die Nektarsekretion von Baumwolle (en russe ; Apic. Abstr. I 72/6t). Pchelozodstwo, 36, 25-38.

Fenoyer L. A., т9г6. Environmental influences on nectar secretion. Bot. Gaz., 63, 249-265.

Kierulf 13., 1957. Is it possible to predict the heather flow? (en norvégien). Nord. Bitidsk., 9, 42-44 
Kuraishi S., Muir R. M., I963. Mode of action of growth retarding chemicals. Plant Physiol., 38, I9-29.

Liebig J. Von, i $8+6$. Lin Agriculturchemie ( 6 éd.).

Lütтge O., ı962. Über die Zuzammensetzung des Nektars und den Mechanismus seiner Sekretion. II. Der kationengehalt des Nektars und die Bedeutung des Verhältnisses $\mathrm{Mg}^{++} \mathrm{Ca}^{++}$im Drüsengewebe für die Sekretion. Planla, 59, I08-1 I4.

Maksymiuk 1., r960. The nectar secretion of linden Tilia cordata miLl. at Reserve Obrozyska near Muzyna (Carpathians) (en polonais). Pczczel. Zeszel. Nauk., 4, 105-1 25.

Matile P., 1956. Über den Stoffwechsel und die Auxinabhängigkeit der Nektarsekretion. Schacei: Bot. Ges. Ber., 66, 237-266.

Maurizio A., 1954. Untersuchungen über die Nektarsekretion einiger polyploider Kulturpfanzen. Schieiz. Ges. Vererbi. Jahresb., 29, 340-346.

Meyer 13. S., Anderson D. B., 1952. Plant Physiology, and ed., 335-365, Van Yostrand, New York.

MOFFETT J. O., PARKer R. L., 1953. Relation of weather factors to nectar How in honey production. Kansas Agr. Exp. Sta., Bull., 74.

Monakova X., Chebotnikova K. M., i955. Increasing the nectar production of honey plants by means of various fertilizers (en russe ; Apic. Abstr. 245/57). Pchelovodstoo, 8, 44-46.

Oertel E., I93I. Hydrogen-ion concentration of soils and its relation to the importance of white clover as a honey plant. J. Econ. Ent., 24, 627-632.

Pentersen M. W., I953. Environmental factors affecting nectar secretion and seed production in alfalfa. Agron. J., 45, 359-36r.

Pedersen M. W., 1953 a. Seed production in alfalfa as related to nectar production and honeybee visitation. Bot. Gaz., 115, 1 $29^{-1} 3^{8}$.

PEDERSEN M. W., i957. Effects of thimning an established stand, of nitrogren supply, and of temperature on nectar secretion in alfalfa. Bot. Gas., 119, г19-122.

Percival M. S., I946. Observations on the flowering and nectar secretion of Rubus fructicusus (AGg.). Neqe Phytol., 45, I I I-1 23 .

PLASS F., I952. Versuche zur Festsellung des Linfusses der Mineraldungung auf die Nelitarabsonderings der Obstgenochse. Bienenzucht, 5, 270-27 I.

Ponomareva 1. G., Bukhareva G. A., I958. The pollination of lucerne by honeybees (en rusie; Apic. Alsstr. 415/59). Pchelovodstvo, 3-4, 2I-24.

Ryle M., 1954. The influence of nitrogen, phosphate, and potash on the secretion of nectar. J. Agr. Sci., 44, 400-407; 408-419.

Schöntag A., 1952. Der Finfluss von Mineralstoffen auf die Nektarabscheidung durch die PHanzen. Niturwiss, 39, 304-305.

Shaw F., Bourne A., Migliorani R., i 957. The effects of various levels of fertilizers on the growth and nectar secretion of snapdragons (Antirrhinum majus). Glean. Bee Cult., 85, 598-599.

Shuel R. W., 1952. Some factors affecting nectar yield in red clover. Plant Physiol., 27, 95-1 10.

Shuel R. W., Sinvas J. A., I953. The influence of soil physical condition during the flowerinz period on nectar production in snapdragon. Plant Physiol., 28, 645-651.

Shuel R. W., 1955. Nectar secretion in relation to nitrogen supply, nutritional status, and growth of the plant . Canad. J. Agric. Sci., 35, I24-1.38.

Shuel R. W., I955 a. Nectar secretion. Amer. Bee J., 95, 229-2.34.

Sinel R. W., I957. Some aspects of the relation between nectar secretion and nitrogen, phosphorus, and potassium nutrition. Canad. J. Plant Sci., 37, 220-236.

SHuel R. W., I959. Studies of nectar secretion in excised flowers II. Influence of certain growth regulators and enzyme inhibitors. Canad. J. Bot., 37, i I67-1 I 80.

Shuks R. IV., I96r. The influence of calcium and magnesium supply on nectar production in red clover and snapdragon. Canad. J. Plant Sci, 41, 50-58.

Tsciudin E., I92r. Nectar secretion affected by altitude. Gleanings Bee Cull., 49, 100.

Ukkelberg B., ig60. Temperature and the heather flow (en norvégien).

UkkelberG B., I960. Temperature and the heather flow (en norvérien; Apic. Abstr. 273/60). Birokteren, 76, 42-43.

WyKes G. R., I952. The infuence of variations in the supply of carbohydrates on the process of nectar, secretion. New Phytol., 51, 294-300.

Ziegler H., LutTge U., I 955. Über die Resorption von C14-Glutaminsäure durch sezernierende Jektarien. Naturwiss., 42, 259-260.

ZimmermanN M., I953. Pepierchromatographische Untersuchungen über dic pflanzliche Zuckersekretion Ber. Scheweiz. Bot. (jes., 63, 402-420. 